\title{
Witnessing nonclassical multipartite states
}

\author{
A. Saguia $\{$ C. C. Rulli, Thiago R. de Oliveira, and M. S. Sarandy \\ Instituto de Física, Universidade Federal Fluminense, \\ Av. Gal. Milton Tavares de Souza s/n, Gragoatá, 24210-346, Niterói, RJ, Brazil.
}

(Dated: November 17, 2018)

\begin{abstract}
We investigate a witness for nonclassical multipartite states based on their disturbance under local measurements. The witness operator provides a sufficient condition for nonclassicality that coincides with a nonvanishing global quantum discord, but it does not demand an extremization procedure. Moreover, for the case of $Z_{2}$-symmetric systems, we rewrite the witness in terms of correlation functions so that classicality is found to necessarily require either vanishing magnetization in the invariant axis or isotropy of the two-point function in the transverse spin plane. We illustrate our results in quantum spin chains, where a characterization of factorized ground states (with spontaneously broken $Z_{2}$ symmetry) is achieved. As a by-product, the witness will also be shown to indicate a second-order quantum phase transitions, which will be illustrated both for the $X Y$ and Ashkin-Teller spin chains.
\end{abstract}

PACS numbers: 03.65.Ud, 03.67.Mn, 75.10.Jm

\section{INTRODUCTION}

The characterization of quantum correlated multipartite systems has been a central topic in quantum information theory (see, e.g., Refs. [1 [3] for recent discussions). Such systems are described by quantum states that cannot be represented by joint probability distributions. Remarkably, the existence of quantum correlations may occur even in the absence of entanglement and still be a resource for a number of quantum information applications (see, e.g., Refs. [4-8]). Measures of quantum correlations provide then a valuable tool to recognize useful quantum states for a certain task. In this context, an important measure is given by quantum discord (QD) [9]. It arises as a difference between two expressions for the total correlation in a bipartite system (as measured by the mutual information), which are classically equivalent but distinct in the quantum regime. QD has been generalized to the multipartite scenario by different approaches [10-13]. Unfortunately, both bipartite and multipartite versions of QD involve extremization procedures, which makes their computation viable only for very small systems or for particular classes of states.

In this context, a possible strategy to identify composite systems that do not exhibit purely classical correlations is to look for a witness operator, namely, an observable able to detect the presence of nonclassical states. A witness $\mathcal{W}$ is defined here as a Hermitian operator whose norm $\|\mathcal{W}\|$ is such that, for all nonclassical states, $\|\mathcal{W}\|>0$, with a convenient norm measure adopted. Therefore, $\|\mathcal{W}\|>0$ is a sufficient condition for nonclassicality (or, equivalently, a necessary condition for classicality). Several investigations for establishing conditions to witness bipartite nonclassical states have recently been carried out [14 17]. Our aim here

\footnotetext{
* amen@if.uff.br
}

is to provide a systematic approach to obtaining a witness for nonclassicality in multipartite states, which will be based on their disturbance under local measurements. In turn, such a witness will naturally detect states with a nonvanishing multipartite QD. As an illustration, we will consider arbitrary $Z_{2}$-symmetric states, where the witness operator can be conveniently rewritten in terms of correlation functions so that classicality is found to require either vanishing magnetization in the $Z$ direction or isotropy of the two-point function in the $\mathrm{XY}$ spin plane. In particular, we will apply such results in quantum spin chains, where it will be shown that $\|\mathcal{W}\|$ exhibits a completely different behavior in the cases of $Z_{2}$-symmetric states and states with spontaneous symmetry breaking (SSB). In the latter case, a characterization of nontrivial factorized ground states (with $Z_{2} \mathrm{SSB}$ ) will be achieved. As a by-product, the witness will also be shown to exhibit a nonanalytical behavior at second-order quantum phase transitions. This nonanalyticity will be revealed by the first derivative of $\|\mathcal{W}\|$ and will be used as a tool to characterize second-order quantum critical points in both the XY and Ashkin-Teller spin chains.

\section{WITNESS FOR NONCLASSICAL STATES}

Let us begin by considering a composite system in an $N$-partite Hilbert space $\mathcal{H}=\mathcal{H}_{A_{1}} \otimes \mathcal{H}_{A_{2}} \otimes \cdots \otimes \mathcal{H}_{A_{N}}$. The system is characterized by quantum states described by density operators $\rho \in \mathcal{B}(\mathcal{H})$, where $\mathcal{B}(\mathcal{H})$ is the set of bound, positive-semidefinite operators acting on $\mathcal{H}$ with trace given by $\operatorname{Tr} \rho=1$. In order to define a multipartite classical state, we will generalize the idea of nondisturbance under projective measurements introduced for bipartite states in Ref. [18]. Indeed, we will denote a set of local von Neumann measurements as $\left\{\Pi_{j}\right\}=\left\{\Pi_{A_{1}}^{i_{1}} \otimes \cdots \Pi_{A_{N}}^{i_{N}}\right\}$, with $j$ denoting the index string $\left(i_{1} \cdots i_{N}\right)$. Then, after a non-selective measurement, the 
density operator $\rho$ becomes

$$
\Phi(\rho)=\sum_{j} \Pi_{j} \rho \Pi_{j} .
$$

This operation can then be used to define a classical state.

Definition. If there exists any measurement $\left\{\Pi_{j}\right\}$ such that $\Phi(\rho)=\rho$ then $\rho$ describes a classical state under von Neumann local measurements.

Therefore, it is always possible to find out a local measurement basis such that a classical state $\rho$ is kept undisturbed. In this case, we will denote $\rho \in \mathcal{C}^{N}$, where $\mathcal{C}^{N}$ is the set of $N$-partite classical states. Observe that such a definition of classicality coincides with the vanishing of global QD [11]. Note also that $\Phi(\rho)$ by itself is a classical state for any $\rho$, namely, $\Phi(\Phi(\rho))=\Phi(\rho)$. Hence, $\Phi(\rho)$ can be interpreted as a decohered version of $\rho$ induced by measurement. A witness for nonclassical states

can be directly obtained from the observation that the elements of the set $\left\{\Pi_{j}\right\}$ are eigenprojectors of $\rho$. This can be shown by the theorem below (see also Ref. [18]).

Theorem $1 \rho \in \mathcal{C}^{N} \Longleftrightarrow\left[\rho, \Pi_{j}\right]=0(\forall j)$, with $\Pi_{j}=$ $\Pi_{A_{1}}^{i_{1}} \otimes \cdots \Pi_{A_{N}}^{i_{N}}$ and $j$ denoting the index string $\left(i_{1} \cdots i_{N}\right)$.

Proof. If $\rho \in \mathcal{C}^{N}$ then $\Phi(\rho)=\rho$. Then, similarly as in Ref. [18], a direct evaluation of $\sum_{j}\left[\rho, \Pi_{j}\right]\left[\rho, \Pi_{j}\right]^{\dagger}$ yields $\sum_{j}\left[\rho, \Pi_{j}\right]\left[\rho, \Pi_{j}\right]^{\dagger}=\Phi\left(\rho^{2}\right)-\rho^{2}$. However, $\Phi(\rho)=\rho$ also implies that $\Phi\left(\rho^{2}\right)=\rho^{2}$. Therefore,

$$
\sum_{j}\left[\rho, \Pi_{j}\right]\left[\rho, \Pi_{j}\right]^{\dagger}=0
$$

Hence, $\rho \in \mathcal{C}^{N} \Longrightarrow\left[\rho, \Pi_{j}\right]=0$. On the other hand, if $\left[\rho, \Pi_{j}\right]=0$ then $\left\{\Pi_{j}\right\}$ provides a basis of eigenprojectors of $\rho$. Then, from the spectral decomposition, we obtain

$$
\rho=\sum_{j} p_{j} \Pi_{j}=\sum_{i_{1}, \cdots, i_{N}} p_{i_{1}, \cdots, i_{N}} \Pi_{A_{1}}^{i_{1}} \otimes \cdots \Pi_{A_{N}}^{i_{N}}
$$

which immediately implies that $\Phi(\rho)=\rho$. Hence $\left[\rho, \Pi_{j}\right]=0 \Longrightarrow \rho \in \mathcal{C}^{N}$.

We can now propose a necessary condition to be obeyed for arbitrary multipartite classical states.

Theorem 2 Let $\rho$ be a classical state and $\rho_{A_{i}}$ the reduced density operator for the subsystem $A_{i}$. Then $\left[\rho, \rho_{A_{1}} \otimes \cdots \otimes \rho_{A_{N}}\right]=0$.

Proof. From theorem 1, if $\rho \in \mathcal{C}^{N}$ then the spectral decomposition of $\rho$ yields Eq. (3). Therefore,

$$
\rho_{A_{i}}=\sum_{n} p_{n} \Pi_{A_{i}}^{n}
$$

Hence, by direct evaluation, we obtain that $\left[\rho, \rho_{A_{1}} \otimes \cdots \otimes \rho_{A_{N}}\right]=0$.
Observe that, given a composite multipartite state $\rho$, it is rather simple to evaluate the commutator $\left[\rho, \rho_{A_{1}} \otimes \cdots \otimes \rho_{A_{N}}\right]$, with no extremization procedure as usually required by QD computation. From the necessary condition for classical states above, we can define a witness for nonclassicality by the norm of the operator $\left[\rho, \rho_{A_{1}} \cdots \rho_{A_{N}}\right]$. Indeed, if $\rho \in \mathcal{C}^{N} \Longrightarrow\|\mathcal{W}\|=0$, where

$$
\mathcal{W}=\left[\rho, \rho_{A_{1}} \otimes \cdots \otimes \rho_{A_{N}}\right]
$$

Therefore, $\|\mathcal{W}\|>0$ is sufficient for nonclassicality.

For concreteness, we will take $\|\mathcal{W}\|$ as defined by the trace norm, namely,

$$
\|\mathcal{W}\|=\operatorname{Tr} \sqrt{\mathcal{W} \mathcal{W}^{\dagger}}
$$

The witness $\|\mathcal{W}\|$ provides a sufficient - but not necessary - condition for nonclassicality. As a simple example, consider a two-qubit system $A B$ prepared in a Bell state $|\psi\rangle=(|00\rangle+|11\rangle) / \sqrt{2}$, where $\{|0\rangle,|1\rangle\}$ denotes the computational basis for both qubits $A$ and $B$. The reduced density operators for each qubit are given by maximally mixed states, with $\rho_{A} \otimes \rho_{B}$ proportional to the identity operator. Therefore, by taking $\rho=|\psi\rangle\langle\psi|$, we have that $\left[\rho, \rho_{A} \otimes \rho_{B}\right]=0$, even though $\rho$ describes a maximally entangled state. However, as we will show below, such a witness can be a very useful tool to investigate nonclassicality in other (non-maximally entangled) classes of quantum correlated states in many-body systems.

\section{NONCLASSICAL STATES IN BIPARTITE SYSTEMS WITH $Z_{2}$ SYMMETRY}

As an application of the nonclassicality witness, let us translate the witness operator $\|\mathcal{W}\|$ in terms of correlation functions and magnetization in quantum spin systems. To this aim, we will consider an interacting pair of spins- $1 / 2$ particles in a chain, which is governed by an arbitrary Hamiltonian $H$ that is both real and exhibits $Z_{2}$ symmetry, i.e. invariance under $\pi$-rotation around a given spin axis. By taking this spin axis as the $z$ direction, this implies the commutation of $H$ with the parity operator $\bigotimes_{i=1}^{N} \sigma_{i}^{3}$, where $N$ denotes the total number of spins and $\sigma_{i}^{3}$ is the Pauli operator along the $z$-axis at site $i$. From this symmetry, the two-spin reduced density matrix at sites labelled by $i$ and $j$ in the basis $\{|\uparrow \uparrow\rangle,|\uparrow \downarrow\rangle,|\downarrow \uparrow\rangle,|\downarrow \downarrow\rangle\}$, with $|\uparrow\rangle$ and $|\downarrow\rangle$ denoting the eigenstates of $\sigma^{3}$, will be given by an $X$-state, reading

$$
\rho_{A B}=\left(\begin{array}{cccc}
a & 0 & 0 & f \\
0 & b_{1} & z & 0 \\
0 & z & b_{2} & 0 \\
f & 0 & 0 & d
\end{array}\right)
$$


In terms of spin correlation functions, these elements can be written as

$$
\begin{aligned}
a & =\frac{1}{4}\left(1+G_{z}^{i}+G_{z}^{j}+G_{z z}^{i j}\right), \quad z=\frac{1}{4}\left(G_{x x}^{i j}+G_{y y}^{i j}\right) \\
b_{1} & =\frac{1}{4}\left(1+G_{z}^{i}-G_{z}^{j}-G_{z z}^{i j}\right), \quad f=\frac{1}{4}\left(G_{x x}^{i j}-G_{y y}^{i j}\right) \\
b_{2} & =\frac{1}{4}\left(1-G_{z}^{i}+G_{z}^{j}-G_{z z}^{i j}\right), \\
d & =\frac{1}{4}\left(1-G_{z}^{i}-G_{z}^{j}+G_{z z}^{i j}\right)
\end{aligned}
$$

where $G_{z}^{k}=\left\langle\sigma_{z}^{k}\right\rangle(k=i, j)$ is the magnetization density at site $k$ and $G_{\alpha \beta}^{i j}=\left\langle\sigma_{\alpha}^{i} \sigma_{\beta}^{j}\right\rangle(\alpha, \beta=x, y, z)$ denote twopoint spin-spin functions at sites $i$ and $j$, with the expectation value taken over the quantum state of the system. Note that, in case of translation invariance, which will be assumed here for simplicity, $G_{z}^{k}=G_{z}^{k^{\prime}} \equiv G_{z}\left(\forall k, k^{\prime}\right)$ and, therefore, $b_{1}=b_{2} \equiv b$. In order to evaluate the witness in terms of the magnetization density and spinspin correlation functions, we compute the commutator $\mathcal{W}=\left[\rho, \rho_{A} \otimes \rho_{B}\right]$, yielding

$$
\mathcal{W}=\left(\begin{array}{cccc}
0 & 0 & 0 & k \\
0 & 0 & 0 & 0 \\
0 & 0 & 0 & 0 \\
-k & 0 & 0 & 0
\end{array}\right)
$$

with $\left.k=f\left[(b+d)^{2}-(a+b)^{2}\right)\right]$. Therefore

$$
\|\mathcal{W}\|=\frac{1}{2}\left|\left\langle\sigma_{x}^{i} \sigma_{x}^{j}\right\rangle-\left\langle\sigma_{y}^{i} \sigma_{y}^{j}\right\rangle\right|\left|\left\langle\sigma_{z}\right\rangle\right| .
$$

Therefore, for translation invariant systems with $Z_{2}$ symmetry, the necessary conditions for a state be classical are either vanishing magnetization in the $z$ spin direction or isotropy in the $X Y$ spin plane, i.e.,

$$
\left\langle\sigma_{z}\right\rangle=0 \text { or }\left\langle\sigma_{x}^{i} \sigma_{x}^{j}\right\rangle=\left\langle\sigma_{y}^{i} \sigma_{y}^{j}\right\rangle .
$$

Hence, the violation of the condition established in Eq. (11) is sufficient for the nonclassicality of the quantum state.

\section{FACTORIZED GROUND STATES IN THE XY MODEL}

As an example of a $Z_{2}$-symmetric system, let us consider a XY spin- $1 / 2$ chain, which is governed by the following Hamiltonian

$H=-\sum_{i=0}^{N-1}\left\{\frac{J}{2}\left[(1+\gamma) \sigma_{i}^{x} \sigma_{i+1}^{x}+(1-\gamma) \sigma_{i}^{y} \sigma_{i+1}^{y}\right]+h \sigma_{i}^{z}\right\}$,

with $N$ being the number of spins in the chain, $\sigma_{i}^{m}$ the $i$-th spin Pauli operator in the direction $m=x, y, z$ and periodic boundary conditions assumed. For the (dimensionless) anisotropy parameter $\gamma \rightarrow 0$, the model reduces to the XX model whereas for all the interval $0<\gamma \leq 1$ it belongs to the Ising universality class, reducing to the transverse field Ising model at $\gamma=1$. The parameter $h$ is associated with the external transverse magnetic field. In the thermodynamical limit, for $\lambda \equiv J / h=1$, a secondorder quantum critical line takes place for any $0<\gamma \leq 1$.

The exact solution of the XY model is well known [19, 20]. The Hamiltonian (12) can be diagonalized via a Jordan-Wigner map followed by a Bogoliubov and Fourier transformation. Given the $Z_{2}$ symmetry, the twospin reduced density operator can be written as

$$
\rho=\frac{1}{4}\left[I \otimes I+\sum_{i=1}^{3}\left(c_{i} \sigma^{i} \otimes \sigma^{i}\right)+c_{4}\left(I \otimes \sigma^{3}+\sigma^{3} \otimes I\right)\right],
$$

where, in terms of the parametrization given in Eq. (17), we have $c_{1}=2 z+2 f, c_{2}=2 z-2 f, c_{3}=a+d-2 b$, and $c_{4}=a-d$ (with $\left.b_{1}=b_{2} \equiv b\right)$. Due to the fact that the system is translationally invariant, the reduced state (13) depends only on the distance between the spins. We will consider here nearest-neighbor pairs. The magnetization density $G_{z}=\left\langle\sigma_{z}\right\rangle$ and the two-point functions $G_{\alpha \beta}^{i, i+1}=$ $\left\langle\sigma_{\alpha}^{i} \sigma_{\beta}^{i+1}\right\rangle$ can be directly obtained from the exact solution of the model [19, 20].

In the ordered ferromagnetic phase $(\lambda>1)$, a finite magnetization $\left\langle\sigma_{i}^{x}\right\rangle$ (in the coupling direction) emerges, breaking the $Z_{2}$ symmetry of the Hamiltonian for an infinite chain. This is possible because, at the critical point, the ground state becomes two-fold degenerated, exhibiting opposites values of $\left\langle\sigma_{i}^{x}\right\rangle$ (denoted as $|+m\rangle$ and $|-m\rangle$ ). Naturally, a superposition of the two ground states is also a ground state, which could then preserve the $Z_{2}$ symmetry. However, in the thermodynamic limit, these two degenerated ground states are not connected by local unitaries. Thus, to go from one to the other, one needs to flip all the spins at the same time, which is both a highly non-local and energy costly operation. Therefore, a small external perturbation will always pick up one of the two states, and the system will be "frozen" at it. The use of a superposition that preserves the symmetry could be justified in the case of a finite system, where the system, if highly isolated, could exist in such state. Another possibility would be to consider an equal mixture of the two ground states, which could be reasonable in the case one prepares a system at some temperature $T$ and cools it down to the limit of $T \rightarrow 0$. This is a $Z_{2}$-symmetric state called thermal ground state. Note that, since the thermodynamic properties depend only on the energy spectrum (and not on the eigenstates), all choices of ground states will be equivalent. However, classical and quantum correlations do depend on the eigenstates and, therefore, the symmetric and broken choices of ground states may exhibit different pattern of correlations [21 24].

Remarkably, the ground state with SSB exhibits a factorization (product state) point at

$$
\gamma^{2}+\left(\frac{1}{\lambda}\right)^{2}=1
$$


This point identifies a change in the behavior of the correlation functions decay, which pass from monotonically to an oscillatory decay [20]. Besides, it has been realized that, at this point, the product ground state is two-fold degenerated even for finite systems [22 24].

In order to apply the witness $\|\mathcal{W}\|$ in the XY model, we will first consider the case of the thermal $\left(Z_{2}\right.$-symmetric) ground state, for which the reduced density matrix is given by Eq. (13). For this case, a plot of $\|\mathcal{W}\|$ as a function of $\lambda$ is provided in Fig. 1 for $\gamma=0.6$. Observe that this plot shows that the only possible classical state appears for $\lambda=0$. Indeed, this point corresponds to an infinite transverse magnetic field applied, which leads the system to a product state, with all spins individually pointing in the $z$ direction. Concerning the factorization point given in Eq. (14), it is absent for the thermal state, since the statistical mixture of non-orthogonal product states exhibits nonvanishing quantum correlations [23].

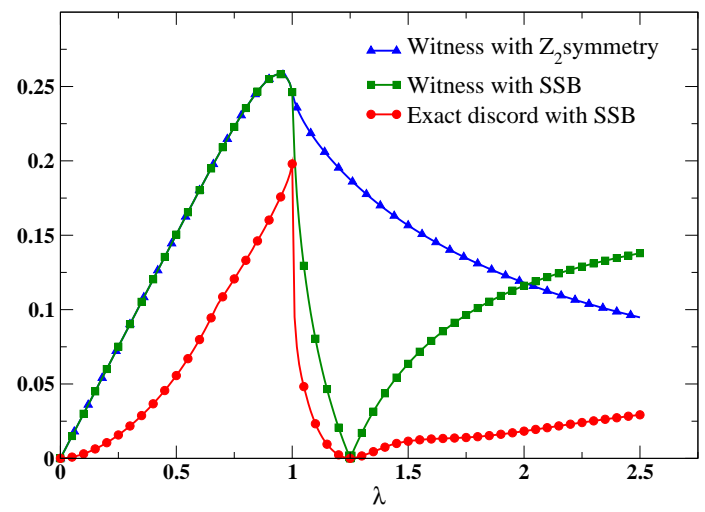

FIG. 1. (Color online) Witness $\|\mathcal{W}\|$ and exact value of QD for a pair of spins in both symmetric and broken ground states of the XY spin-1/2 infinite chain as a function of $\lambda$ for $\gamma=0.6$.

On the other hand, if SSB is taken into account, which must be the case in the thermodynamic limit, this picture dramatically changes. Indeed, in the broken case, the two-spin reduced density matrix is more complicated and reads

$$
\rho_{A B}=\left(\begin{array}{cccc}
a & p & p & f \\
p & b & z & q \\
p & z & b & q \\
f & q & q & d
\end{array}\right)
$$

where the functions $p(\lambda, \gamma)$ and $q(\lambda, \gamma)$ are given by

$$
\begin{aligned}
& p(\lambda, \gamma)=\left\langle\sigma_{x}^{i}\right\rangle+\left\langle\sigma_{x}^{i} \sigma_{z}^{i+1}\right\rangle, \\
& q(\lambda, \gamma)=\left\langle\sigma_{x}^{i}\right\rangle-\left\langle\sigma_{x}^{i} \sigma_{z}^{i+1}\right\rangle .
\end{aligned}
$$

The evaluation of these functions have been detailed discussed in Ref. [21], where tight lower and upper bounds for $p(\lambda, \gamma)$ and $q(\lambda, \gamma)$ are obtained. In our plots, either of such bounds essentially yields the same curves, which led us to keep the results produced with the lower bound. Concerning the results for $\|\mathcal{W}\|$, we can compare the plots for the thermal ground state and the ground state with SSB also in Fig. 1. Observe that, in the case with $\mathrm{SSB}$, the only point for which $\|\mathcal{W}\|=0$ is $\lambda=1.25$. Indeed, this corresponds to a classical state, as can be confirmed by the exact computation of the symmetric QD. Remarkably, this classical state is associated with one of two doubly-degenerated factorized (fully product) ground state, which appears as a consequence of the $Z_{2}$ SSB. This characterization of factorized ground states through $\|\mathcal{W}\|=0$ can be numerically confirmed in Fig. 2, where $\|\mathcal{W}\|$ is plotted for a number of different values of the parameter $\gamma$. These unique classical points for the XY model are then clearly revealed by the witness evaluation. Another aspect of the witness observed from Figs. 1 and 2 is that it exhibits nonanalyticity in its first derivative at the second-order quantum critical point $\lambda=1$. This phenomenon occurs both for the symmetric and broken ground states, which promotes $\|\mathcal{W}\|$ to a simple useful tool also to detect quantum phase transitions.

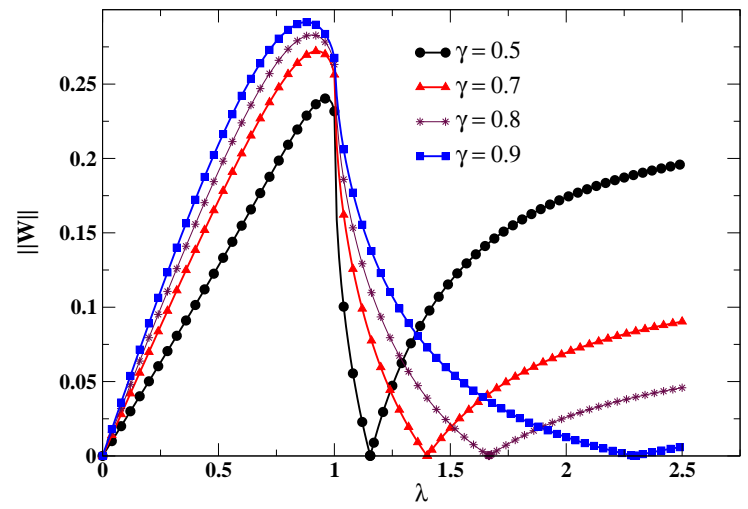

FIG. 2. (Color online) Witness $\|\mathcal{W}\|$ for a pair of spins in the ground state with $\mathrm{SSB}$ of the XY spin- $1 / 2$ infinite chain as a function of $\lambda$ for several values of the parameter $\gamma$.

\section{QUANTUM CORRELATED STATES IN THE ASHKIN-TELLER MODEL}

The Ashkin-Teller model has been introduced as a generalization of the Ising spin-1/2 model to investigate the statitiscs of lattices with four-state interacting sites [25]. It exhibits a rich phase diagram [26] and has recently attracted a great deal of attention due to several applications, e.g., nonabelian anyon models 27] and orbital current loops in $\mathrm{CuO} 2$-plaquettes of high-Tc cuprates [28]. The Hamiltonian for the quantum Ashkin-Teller model in one-dimension for a chain with $M$ sites is given by

$$
\begin{gathered}
H_{A T}=-J \sum_{j=1}^{M}\left(\sigma_{j}^{x}+\tau_{j}^{x}+\Delta \sigma_{j}^{x} \tau_{j}^{x}\right) \\
-J \beta \sum_{j=1}^{M}\left(\sigma_{j}^{z} \sigma_{j+1}^{z}+\tau_{j}^{z} \tau_{j+1}^{z}+\Delta \sigma_{j}^{z} \sigma_{j+1}^{z} \tau_{j}^{z} \tau_{j+1}^{z}\right),
\end{gathered}
$$


where $\sigma_{j}^{\alpha}$ and $\tau_{j}^{\alpha}(\alpha=x, y, z)$ are independent Pauli spin- $1 / 2$ operators, $J$ is the exchange coupling constant, $\Delta$ and $\beta$ are (dimensionless) parameters, and periodic boundary conditions (PBC) are adopted, i.e., $\sigma_{M+1}^{\alpha}=\sigma_{1}^{\alpha}$ and $\tau_{M+1}^{\alpha}=\tau_{1}^{\alpha}(\alpha=x, y, z)$. The Ashkin-Teller model is $Z_{2} \otimes Z_{2}$-symmetric, with the Hamiltonian commuting with the parity operators

$$
\mathcal{P}_{1}=\otimes_{j=1}^{M} \sigma_{j}^{x} \quad \text { and } \quad \mathcal{P}_{2}=\otimes_{j=1}^{M} \tau_{j}^{x} .
$$

Therefore, the eigenspace of $H_{A T}$ can be decomposed into four disjoint sectors labelled by the eigenvalues of $\mathcal{P}_{1}$ and $\mathcal{P}_{2}$. We then numerically diagonalize $H_{A T}$ (via power method) to evaluate the witness $\|\mathcal{W}\|$, which provides a sufficient condition for the absence of $Z_{2} \otimes Z_{2}$-symmetric classical states for finite values of $\beta$ and $\Delta$. We illustrate this result by considering different configurations of multipartite states. More specifically, we choose a spin quartet, namely, a block of $L=4$ particles described by spin operators $\bigotimes_{k=j}^{j+1} \hat{\sigma}_{k} \hat{\tau}_{k}$, and respective generalization for a spin octet. For such cases, the absence of classical states for any finite $\Delta$ is displayed in Fig. 3 for $\beta=1$ in a chain with $N=16$ particles. This is confirmed in the inset of Fig. 3, where the exact behavior of global QD is exhibited.

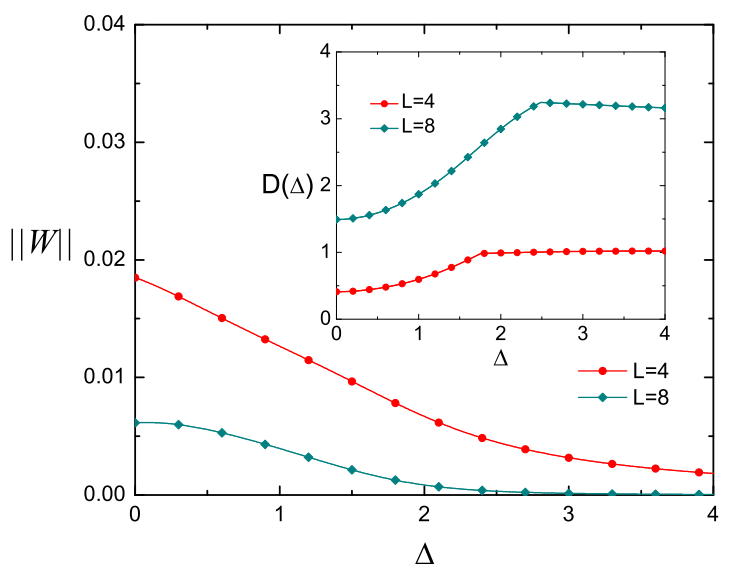

FIG. 3. (Color online) Witness $\|\mathcal{W}\|$ as a function of $\Delta$ for several $L$-spin multipartite states in the Ashkin-Teller model for $\beta=1$ in a chain with $N=16$ spin particles.

Moreover, besides sufficent for identifying no classical states in the thermal $\left(Z_{2} \otimes Z_{2}\right.$-symmetric) ground state of the Ashkin-Teller chain, the witness $\|\mathcal{W}\|$ can also be applied to identify second-order quantum phase transitions driven by the parameter $\beta$. We illustrate this result by plotting $\|\mathcal{W}\|$ for a quartet in Fig. 4 as a function of $\beta$ for $\Delta=3$. From this plot, a second-order critical point can be readout through a minimum in the derivative of $\|\mathcal{W}\|$ (see inset in Fig. 4), which gets pronounced as the length of the chain is increased (we observe that the maximum appearing in the plot for $d\|\mathcal{W}\| / d \beta$ does not indicate any transition, since it does not exhibit pronunciation as we increase the size of the chain). This pronounced minimum, which is a typical precursor of the nonanalyticity at the thermodynamic limit, occurs for $\beta=0.61$, which is compatible with the quantum phase diagram in Ref. [26]. A further transition would be expected to occur near $\beta=1.5$. This critical point is not apparent from $\|\mathcal{W}\|$, at least for chains up to $N=20$ spins. Indeed, this second critical point is usually harder to characterize (see, e.g., characterization with entanglement in Ref. 29] and with global QD in the upper insets of Fig. 4, where pronunciation is subtle).

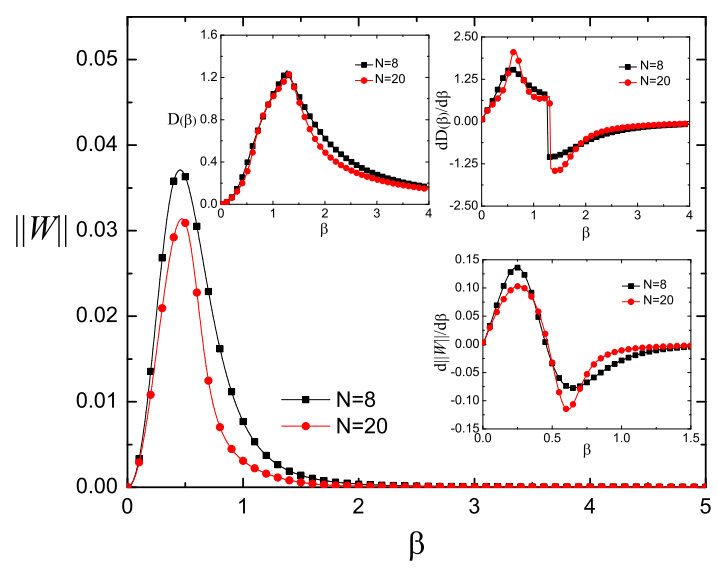

FIG. 4. (Color online) Witness $\|\mathcal{W}\|$ as a function of $\beta$ for a quartet in the Ashkin-Teller model for $\Delta=3$ in chains with $N=8$ and $N=20$ spin particles.

\section{CONCLUSION}

In summary, we have proposed a multipartite witness for nonclassical states based on their disturbance under local measurements. This witness provides a sufficient condition for nonclassicality that is in agreement with a nonvanishing global QD. As an illustration, we derived the necessary conditions for classicality in terms of correlation functions for $Z_{2}$-symmetric density operators and apply the witness for both the XY and Ashkin-Teller chains. Remarkably, the witness detects the factorized ground state of the XY chain as its unique classical state (for finite value of $\lambda$ and $\gamma$ ) as the SSB was taken into account. Then, in this example, $\|\mathcal{W}\|$ turns out to be not only sufficient but also necessary for nonclassicality. Moreover, the witness was also shown to be able to identify second-order quantum phase transitions through a nonanalyticity in the first derivative of $\|\mathcal{W}\|$ for both $\mathrm{XY}$ and Ashkin-Teller chains. Even though classicality is rare in many-body microscopic systems [15], it is rather useful, as a resource to both quantum information and condensed matter applications, to characterize the existence of nonclassical correlation through simple and direct pro- 
cedures. In particular, the typicality of states with vanishing genuine $n$-partite correlations would be a possible application of our results. The witness $\|\mathcal{W}\|$ may constitute a helpful tool for such kind of characterization.

\section{Acknowledgments}

We acknowledge financial support from the Brazilian agencies $\mathrm{CNPq}$ and FAPERJ. This work was performed as part of the Brazilian National Institute for Science and Technology of Quantum Information (INCT-IQ).
[1] M. Piani, P. Horodecki, and R. Horodecki, Phys. Rev. Lett. 100, 090502 (2008).

[2] A. Acín, R. Augusiak, D. Cavalcanti, C. Hadley, J. K. Korbicz, M. Lewenstein, Ll. Masanes, and M. Piani, Phys. Rev. Lett. 104, 140404 (2010).

[3] C. H. Bennett, A. Grudka, M. Horodecki, P. Horodecki, and R. Horodecki, Phys. Rev. A 83, 012312 (2011).

[4] W. H. Zurek, Phys. Rev. A 67, 012320 (2003).

[5] D. P. DiVincenzo, M. Horodecki, D. W. Leung, J. A. Smolin, and B. M. Terhal, Phys. Rev. Lett. 92, 067902 (2004).

[6] A. Datta, A. Shaji, and C. M. Caves, Phys. Rev. Lett. 100, 050502 (2008).

[7] A. Brodutch and D. R. Terno, Phys. Rev. A 83, 010301(R) (2011).

[8] F. F. Fanchini, M. F. Cornelio, M. C. de Oliveira, and A. O. Caldeira, Phys. Rev. A 84, 012313 (2011).

[9] H. Ollivier and W. H. Zurek, Phys. Rev. Lett. 88, 017901 (2001).

[10] K. Modi, T. Paterek, W. Son, V. Vedral, and M. Williamson, Phys. Rev. Lett. 104, 080501 (2010).

[11] C. C. Rulli and M. S. Sarandy, Phys. Rev. A 84, 042109 (2011).

[12] I. Chakrabarty, P. Agrawal, and A. K. Pati, e-print arXiv:1006.5784 (2010).

[13] M. Okrasa and Z. Walczak, e-print arXiv:1101.6057 (2011)..

[14] B. Bylicka and D. Chruscinski, Phys. Rev. A 81, 062102 (2010).
[15] A. Ferraro, L. Aolita, D. Cavalcanti, F. M. Cucchietti, and A. Acín, Phys. Rev. A 81, 052318 (2010).

[16] B. Dakić, V. Vedral, and C. Brukner, Phys. Rev. Lett. 105, 190502 (2010).

[17] J. Maziero and R. M. Serra, e-print arXiv:1012.3075 (2010).

[18] S. Luo, Phys. Rev. A77, 022301 (2008).

[19] E. Barouch, B. M. McCoy, and M. Dresden, Phys. Rev. A 2, 1075 (1970).

[20] E. Barouch and B. M. McCoy, Phys. Rev. A 3, 786 (1971).

[21] T. R. de Oliveira, G. Rigolin, M. C. de Oliveira, and E. Miranda, Phys. Rev. A 77, 032325 (2008).

[22] S. M. Giampaolo, G. Adesso, and F. Illuminati, Phys. Rev. Lett. 100, 197201 (2008).

[23] L. Ciliberti, R. Rossignoli, and N. Canosa, Phys. Rev. A 82, 042316 (2010).

[24] B. Tomasello, D. Rossini, A. Hamma, and L. Amico, eprint arXiv:1012.4270 (2010).

[25] J. Ashkin and E. Teller, Phys. Rev. 64, 178 (1943).

[26] M. Kohmoto, M. den Nijs, and L. P. Kadanoff, Phys. Rev. B 24, 5229 (1981).

[27] C. Gils, J. Stat. Mech. P07019 (2009).

[28] M. S. Grønsleth, T. B. Nilssen, E. K. Dahl, E. B. Stiansen, C. M. Varma, and A. Sudbø, Phys. Rev. B 79, 094506 (2009).

[29] C. C. Rulli and M. S. Sarandy, Phys. Rev. A 81, 032334 (2010). 